\title{
ASPECTOS PSICOSOCIALES Y ACCIDENTES EN EL TRANSPORTE TERRESTRE
}

\author{
Nelson Morales-Soto1,2,3,a, Daniel Alfaro-Basso1,2,a, Wilfredo Gálvez-Rivero
}

\begin{abstract}
RESUMEN
Los accidentes de tránsito son un problema de salud pública en el Perú, que entre 1998 y 2008 causaron 35596 muertes, Lima es la región más afectada con $61,7 \%$ de los siniestros, su costo anual alcanzó los mil millones de dólares, equivalente a un tercio de la inversión en salud. Los estudios disponibles enfatizan en los protagonistas -conductores, peatones- o en equipos y vías; se han modificado normas e implementado planes de contención de la siniestralidad pero su incidencia persiste. Se plantea la posibilidad de explorar factores conductuales y sociales que podrían tener importancia en la génesis del problema revisando los relacionados con el desorden imperante en el transporte, los comportamientos de conductores y peatones y la permisividad de la sociedad en general, particularmente de la autoridad. Se propone la investigación e intervención multidisciplinaria e intersectorial.
\end{abstract}

Palabras clave: Accidentes de tránsito; Violencia; Conducta social; Salud pública (fuente: DeCS BIREME).

\section{PSYCHOSOCIAL ASPECTS AND ACCIDENTS IN LAND TRANSPORT}

\begin{abstract}
Road traffic accidents are a public health problem in Peru, having caused 35596 deaths in Peru between 1998 and 2008. Lima is the most affected region, presenting $61.7 \%$ of the accidents, the annual cost reached one thousand million dollars, equivalent to a third part of the investment in health. Available studies give emphasis to the protagonists -the drivers, the pedestrians - or to equipment and roads; the laws have been modified and containment plans for accidents have been implemented, but the incidence remains the same. We raise the possibility of exploring behavioral and social factors that could be relevant in the genesis of the problem, revising those related to current disorder in transport, the behaviors of drivers and pedestrians and the permissiveness of society in general particularly of the authority. We propose research and a multidisciplinary and intersectoral intervention.
\end{abstract}

Key words: Traffic, accident; Violence; Social behavior; Public health (source: MeSH NLM).

\section{INTRODUCCIÓN}

La importancia sanitaria, económica y social de los accidentes del transporte terrestre es reconocida a nivel mundial, las lesiones resultantes causan el $2,1 \%$ del total de las muertes y ocupan el undécimo puesto entre las principales causas de muerte ${ }^{(1)}$.

Según el Banco Interamericano de Desarrollo, un millón de personas mueren cada año en las carreteras del mundo y otros 50 millones sufren lesiones. Esta cifra podría incrementarse en $82 \%$ en América Latina para el año 2020 constituyéndose en un problema para su desarrollo pues el costo promedia 1 al $2 \%$ de su PBI (2). En la actualidad, es la primera causa de muerte entre los 15 a los 29 años y la segunda para menores de esa edad siendo los peatones, ciclistas y motociclistas la mitad de las víctimas de carretera ${ }^{(3)}$.
Los accidentes de tránsito no sólo producen daños directos a la salud, también dejan secuelas físicas ${ }^{(4)}$ y psicosociales ${ }^{(5)}$; al igual que en otras emergencias los mayores daños económicos se reportan en los países industrializados, pero la mayor pérdida de vidas ocurre en los países en desarrollo ${ }^{(6)}$. La problemática del transporte va mas allá del accidente de tránsito, se extiende a la vida cotidiana de los ciudadanos $\mathrm{y}$, por ende, a su salud y bienestar.

En el Perú, la Estrategia Sanitaria Nacional de Accidentes de Tránsito, coordinada por la Oficina General de Defensa Nacional del Ministerio de Salud, reporta que entre 1998 y 2008 se produjeron 860102 accidentes de tránsito causando 35596 muertes; Lima fue la región más afectada con $61,73 \%$ de los accidentes en el 2008 ; estos daños constituyen la tercera causa de muerte y su costo anual alcanza los mil millones de dólares, equivalente

\footnotetext{
Facultad de Medicina, Universidad Nacional Mayor de San Marcos. Lima, Perú.

Sociedad Peruana de Medicina de Emergencias y Desastres. Lima, Perú.

Academia Nacional de Medicina. Lima, Perú.

Médico emergenciólogo.
}

Recibido: 14-04-10 Aprobado: 05-06-10 
a un tercio de la inversión en salud. Su importancia ha sido relevada por autoridades municipales y de salud quienes propusieron en el año 2005 intervenciones multidisciplinarias y multisectoriales ${ }^{(7)}$.

Estudios de esta problemática, revisados recientemente por Alfaro-Basso ${ }^{(8)}$, han abordado causas identificadas del accidente tales como el estado del parque automotor, las características del tráfico, la vialidad y la normatividad, tipo y magnitud de daños, entre otros. Se han modificado normas y puesto en práctica iniciativas y "planes piloto", también se ha implementado nuevos controles, pero a pesar de ello no se ha logrado una aceptable reducción de la accidentalidad ni de su morbimortalidad. El problema persiste.

Esto lleva a reflexionar sobre la probable intercurrencia de otros factores insuficientemente esclarecidos que quizá por su cotidianeidad, han dejado de percibirse y que podrian explicar la persistencia del percance, relacionados con modelos de conducta inadecuados pero socialmente aceptados. Surge el interés por identificar la importancia que podrían tener los comportamientos violentos exacerbados por la agitada vida de las metrópolis.

\section{ORDEN}

Una opinión común es que en algunas ciudades el tránsito es caótico y que ello materializa una constante social: el desorden. Conocer en cuánto la informalidad y la transgresión de las normas contribuyen a la creciente inseguridad vial despierta el interés en determinar si hay factores psicosociales que participan como concurrentes en la accidentalidad del transporte terrestre.

El área metropolitana de Lima y el Callao es la de mayor accidentalidad en el país. El Informe Defensorial N. ${ }^{\circ} 137$ del año 2007, menciona que la capital concentra $18,6 \%$ de las muertes y $50,4 \%$ del total de heridos por accidentes de tránsito ${ }^{(9)}$ producidos en sus 12 millones de viajes diarios, $77,3 \%$ suministrados por el transporte público ${ }^{(10)}$.

Se reconoce que en la capital la congestión vehicular y el desorden lentifican exageradamente el tránsito -10 $\mathrm{km} /$ hora en momentos de congestión y $16,8 \mathrm{~km} /$ hora en promedio- alargando el tiempo de viaje en 44,9 minutos en promedio, y agregando un sobrecosto anual de 500 millones de dólares. De no regularse el transporte público se prevé que para el 2025 estos factores promediarán $7,5 \mathrm{~km} /$ hora y 64,8 minutos, respectivamente ${ }^{(10)}$. Esta situación genera tensión y fatiga en conductores y pasajeros, sumándose la contaminación ambiental y acústica que incrementan el malestar y afectan la salud.
Es de interés rescatar y analizar información sobre morbilidad a mediano y largo plazo en conductores o usuarios del transporte en el país.

Un estudio de lesiones en personas jóvenes por accidentes de tránsito en un distrito limeño asoció la mayor incidencia con la exposición, menor tiempo de residencia en la zona, la amplitud de las calles, presencia de comerciantes y el mayor número de vehículos circulantes; los daños ocurrieron con mayor frecuencia entre los 5 y los 14 años de edad y al cruzar las pistas por lugares no autorizados ${ }^{(6)}$.

En el resto del país es noticia recurrente la colisión, en carretera, de grandes buses excedidos de carga y pasajeros -impacto a veces frontal y sin evidencia de maniobra evasiva- o el despiste y precipitación al abismo en zonas agrestes ${ }^{(11,12)}$. Las condiciones de las vías interprovinciales como ausencia de berma central, clima, señalización, o el estado de sueño, cansancio, distracción del conductor, y del trabajo -alta velocidad, competencia- explican muchas colisiones fatales. Se han publicado estudios que revelan las relaciones entre el cansancio, el sueño y los accidentes (13-15), factores que aún no se incluyen en las estadísticas oficiales. La demanda masiva producida en entornos adversos y distantes de los hospitales empeora el pronóstico de las víctimas, aumentando la mortalidad.

En la estadística policial un $33 \%$ de accidentes se adjudica al exceso de velocidad, $28 \%$ a la imprudencia del conductor, $12 \%$ a la imprudencia del peatón, $11 \%$ de los conductores estuvo en estado de ebriedad y sólo en $6 \%$ se identificó falla mecánica del equipo o inadecuada señalización de la vía; en $85 \%$ de casos las causas estuvieron ligadas al hombre, $84 \%$ de los accidentes pudo evitarse tomando medidas preventivas (12). Es conocido que la propensión al accidente se eleva en zonas no frecuentadas por los involucrados.

Es visible la exasperación de los conductores y el mal trato a pasajeros y transeúntes, se compite por el espacio y se buscan atajos para evitar las zonas de mayor congestión, en cuyo afán los atropellos se producen incluso en áreas de circulación peatonal -paraderos, aceras y plazas-y muchas colisiones fatales ocurren en unidades del transporte público que acumulan numerosas infracciones impagas. La magnitud -o complejidad-del problema parece desbordar la capacidad de la autoridad y sus recursos, situación que se acrecentará si no se toman medidas adecuadas, pues se estima que la población capitalina demandará 18 millones de viajes diarios en el $2025^{(10)}$.

La ocurrencia iterativa de siniestros en los que la acción humana aparece como una causa dominante 
debe orientar la investigación. Es evidente que hay un problema de organización que tiene altos costos biológicos y sociales, se requiere conocer si tras los factores mencionados en la estadística oficial subyacen otros componentes individuales -físicos o psicológicos- o sociales y económicos, y determinar cuanta importancia tienen cada uno o en su conjunto en la génesis del evento adverso.

Se menciona que hay multiplicidad de autoridades, superposición de funciones y responsabilidades, así como limitación de recursos, pero la prensa, además, informa que los conductores trabajan excesivo número de horas y que ocurren omisiones sanitarias y laborales; por otro lado, los sobrevivientes de accidentes relatan que los conductores competían arriesgada -cuando no agresivamente- en la pista antes del hecho fatal.

\section{COMPORTAMIENTOS}

Se ha definido como conducción agresiva el manejo de un vehículo motorizado poniendo en peligro -o con probabilidad de poner en peligro- a las personas y las propiedades; caracterizan esta conducta, entre otros: el exceso de velocidad, el zigzaguear, adelantamiento inseguro, no mantener la distancia de seguridad, obstruir el paso de otro vehículo, palabras o gestos obscenos y detención inesperada por pasajeros ${ }^{(16)}$.

En encuestas publicadas en diferentes periódicos de nuestro país los usuarios del transporte público expresan insatisfacción originada por maltratos e inseguridad en el transporte público, así como a pocas rutas. En la entrevista personal a estudiantes universitarios es unánime la referencia al maltrato expresado por conductas agresivas, transgresión a las normas, la competencia por las pistas o por el pasajero, obstrucción intencional del paso a otros vehículos, mal estado de mantenimiento e higiene de las unidades, la tarifa abusiva y el acortamiento antojadizo de la ruta, la estridencia del claxon o el grito como advertencia o agresión, el desorden total del sistema y la falta de respeto a la autoridad.

En el año 2005 la Defensoría de la Policía Nacional reportó que 150 policías femeninas habían sufrido agresiones de conductores violentos, en 84 casos al momento de ser sancionados, algunas fueron embestidas y arrastradas por el vehículo o secuestradas por los choferes ${ }^{(17)}$.

Conductores y pasajeros viven a la defensiva. La elevada frecuencia de robo o asalto en la ciudad, muchos de ellos no denunciados, sobrepasa la posibilidad de acción de la autoridad. En las carreteras también ocurren estos actos, en el 2006 se denunciaron 136 asaltos a vehículos, 74 a buses y en 24 oportunidades durante bloqueos violentos de la vía, el vandalismo causó cinco muertos en el $2007^{(17)}$.

Se reconoce que la violencia en variadas formas es frecuente en la sociedad peruana, particularmente en la familia (18,19). En el 2007 fueron denunciados 87292 casos de violencia familiar, con el agresor ecuánime en 57 mil casos, ebrio en 21 mil, 4 mil estuvieron enfermos o alterados y mil drogados; 39 mil casos ocurrieron en Lima y Callao; 54265 de las agresiones fueron físicas, 25981 psicológicas ${ }^{(17)}$. Se reconoce que podría ocurrir un amplio subregistro.

Los daños por violencia, al igual que los accidentales, tienen gran impacto en la salud. De las 5544 atenciones que la policía registró durante el año 2001 en el servicio de emergencias de un hospital público en Lima, 38,26\% correspondieron a accidentes de tránsito, 24,93\% a otros accidentes y $22,82 \%$ a agresiones, con mayor incidencia entre los 25 a los 49 años ${ }^{(20)}$.

En otros países ocurre algo similar. Se ha reportado un incremento en la tendencia de mortalidad por lesiones intencionales paralelamente a la de accidentes de tránsito -hasta 2,6 veces entre 1999 y el 2000- en la comuna 18 de Cali-Colombia, convirtiéndose en la primera causa de muerte ${ }^{(21)}$.

El $2 \%$ de personas sin aparente trastorno de su salud mental reconocen conductas violentas en su vida, cifra que se incrementa a $24,5 \%$ si hay abuso o dependencia de alcohol y a $19,2 \%$ si hay abuso o dependencia de drogas (22); el consumo de alcohol es percibido por la población como el principal problema que afecta la seguridad pública, 38,7\% a nivel país. Es reconocida la relación entre el consumo de alcohol antes del accidente ${ }^{(23,24)}$, tendencia que, se espera, se atenúe a medida que la población asuma su importancia, acicateada por las recientes penalidades impuestas.

Ponce et al. ${ }^{(25)}$ estudiaron los estilos de comportamiento de conductores del servicio público y vehículos particulares en Lima Metropolitana utilizando el cuestionario de personalidad PSS. Los incluidos en el patrón de conducta tipo $S$-tipo ideal-conducen bajo una percepción y aceptación del riesgo adecuada, realizan una conducción precavida, atenta y responsable, conducen con un alto nivel de seguridad y controlan adecuadamente su conducta; en resumen, son seguros, prudentes, responsables, atentos y precavidos, constituyen el tipo ideal, predomina en los conductores con instrucción superior y aquellos que tienen mayor 
tiempo manejando, fue observado en conductores del servicio interprovincial.

Los inmersos en el patrón de conducta tipo $N$-conducta de alto riesgo y peligrosa- presentan una deficiente percepción del riesgo, son incapaces de reaccionar adecuadamente al "estrés emocional", manifiestan impulsividad, impaciencia, agresividad y tendencias antisociales, poseen escaso control personal, muestran falta de respeto al usuario y a las normas de tráfico, tienden a exteriorizar sus respuestas a la frustración de forma excesiva y poco adecuada. En estos la menor contrariedad -como atascos, retenciones, cruces indebidos- se traduce en irritabilidad y accesos de cólera desproporcionados; en síntesis, su conducta se manifiesta desajustada, de alto riesgo y peligrosa. Fue mayormente observado en conductores particulares, de taxi y microbús más aun si conducían vehículos alquilados o coincidían con problemas familiares o de salud.

El Centro de Investigación y de Asesoría del Transporte Terrestre señala que los vehículos de transporte público estuvieron involucrados en el $57,6 \%$ de los accidentes de tránsito fatales y $61,3 \%$ de las muertes resultantes en Lima Metropolitana ${ }^{(26)}$, los causantes de atropello tenían generalmente más de 35 años, estatura menor a 1,65 m y conducían vehículos de transporte público ómnibus o vehículo de carga con más de 10 años de antigüedad.

Al enfrentarse a situaciones inesperadas y difíciles de resolver como congestión vial, semáforos inoperativos, retrasos en su rutina, o colisiones, podrían aparecer algunos rasgos de personalidad e influir en los accidentes, esto tendría un peso significativo en la propensión a la siniestralidad, se citan la impulsividad, inestabilidad emocional, rebeldía, tendencia a competir, egocentrismo, inmadurez, inseguridad, tensión y ansiedad ${ }^{(24,25)}$.

\section{AUTORIDAD}

Debería respetarse la proporción adecuada entre población, parque automotor y espacio vial. Lima alberga el $60 \%$ del parque vehicular del país pero sólo dispone del $7 \%$ de la red vial nacional. Aunque la accidentalidad es alta se considera que el índice de motorización en el Perú es bajo comparado con otros países de la región, el $80 \%$ de vehículos ingresó usado y algunos alcanzan 20 o más años de antigüedad (12); a pesar de la norma la autoridad permite el ingreso y circulación de estos vehículos.

El parque aumentó de 612249 vehículos en 1989 a 1349510 en el 2005; cada año ingresan al país 75 mil vehículos nuevos y unos 45 mil usados, 13500 de estos no cumplen las normas técnicas que exige la ley y con frecuencia son responsables de los accidentes fatales en Lima, esto coincide además con un incremento en $368 \%$ de infección respiratoria aguda en menores de 5 años de edad en ese período ${ }^{(26)}$.

Para atender los daños del accidente de tránsito se ha creado un seguro obligatorio el cual desde junio de 2002 asumió 320 mil casos que causaron 384 mil heridos y 15500 fallecidos pagando unos 750 millones de soles, $34 \%$ de vehículos no tienen este seguro, a esta transgresión se suma la falsificación de licencias de conducir.

El tránsito de personas y vehículos debe entenderse como un sistema de relaciones dinámico dentro de un ámbito urbano inextensible (27), cuando resulta sobreocupado, como ocurre en Lima, el entorno se muestra hostil en muchos lugares y momentos. Es labor de la autoridad regular los efectos sociales y ambientales ocasionados por el número y características técnicas del parque vehicular ${ }^{(28)}$. Según reporte de la Defensoría del Pueblo el $75 \%$ de comités distritales supervisados no ha aprobado planes de seguridad ciudadana y más del $50 \%$ no ha nombrado secretarios técnicos ${ }^{(29)}$.

El Reglamento Nacional de Tránsito (D.S. N. ${ }^{\circ}$ 033-2001MTC) faculta a la Policía Nacional como la autoridad competente en materia de tránsito terrestre otorgándole competencia de fiscalización en tanto que el Reglamento Nacional de Administración de Transporte (D.S. N. ${ }^{\circ} 009-$ 2004-MTC) le asigna rol de apoyo a dicha autoridad. Los ámbitos de la autoridad del transporte muestran insuficiente interconexión y al igual que en otros espacios sociales la fragmentación y los desacuerdos crean una imagen pública de vacío de reacción, dirección y autoridad ${ }^{(30)}$, la permisividad es aprovechada por el transgresor.

Existen perpetradores y víctimas recurrentes $y$ lugares propicios para el accidente, no pocas veces las causalidades dominan sobre las casualidades particularmente en eventos iterativos ${ }^{(31)}$.

Muchos países reconocen hoy los siniestros viales como una endemia social violenta, no accidental, no originada por el azar ni tampoco como consecuencia del error casual, que es previsible y por lo tanto prevenible, la Organización Mundial de la Salud considera que como el error humano interviene en alta frecuencia la principal solución debería consistir en persuadir a los usuarios de la vía pública a adoptar comportamientos que "no dejen lugar al error" y se adopten medidas apropiadas (1).

El BID ha aprobado un proyecto para mejorar la seguridad vial en la región en atención a compromisos contraídos en la primera Conferencia Mundial de 
Ministros de Seguridad Vial, Moscú 2009, y la Iniciativa Mundial Clinton, en alianza con otras organizaciones como la Organización Mundial de la Salud para crear un sistema regional de información sobre la seguridad vial y promover estudios sobre políticas y prácticas para mejorar la seguridad vial, siendo el Perú parte del proyecto (Banco Interamericano de Desarrollo) ${ }^{(32)}$.

La Estrategia Sanitaria Nacional de Accidentes de Tránsito (4), una de las quince establecidas por el Ministerio de Salud para la prevención y el control de la salud, ha iniciado una investigación sobre las Condiciones de Salud de Conductores de Transporte Urbano de Lima Metropolitana, para determinar los factores que concurren en el incremento de la accidentalidad y para identificar factores individuales $y$ niveles de estrés reciente, así como hábitos nocivos y otras variables físicas de los conductores que puedan influir en la alarmante incidencia de estos accidentes. Este es un tema de gran importancia.

Comparando las proporciones de siniestros en el país, la cifra acumulada de muertes por accidentes de tránsito en el último cuarto de siglo promedia las 70 mil, cifra similar a las causadas por la guerra contra el terrorismo en 20 años y semejante a la ocasionada por el terremoto de 1970 en Huaraz. La accidentalidad, calificada por la OMS en 1974 como un problema de salud pública de suma gravedad ${ }^{(7)}$ se comporta en nuestro medio como un desastre de presentación lenta, de curso sostenido y alta mortalidad, es decir, como una calamidad pública con agravante: es socialmente consentido.

La respuesta sanitaria a la problemática del transporte debe ir más allá de la atención y rehabilitación del accidentado, debe ocuparse de la prevención y enlazarse con los componentes sociales y ambientales. Se debe, además, indagar cuanta influencia tienen los costos descritos en la acumulación de la vulnerabilidad que torna susceptible a la población de menores recursos a nuevos siniestros por su efecto económico adverso inmediato ${ }^{(33)}$ y por sus efectos en la salud y en la familia en el mediano y largo plazo.

Para afrontar el actual problema del transporte público se ha propuesto en un plan maestro el sistema de red vial incluyendo un tren urbano, sistema de buses troncales y alimentadores, y un sistema de administración vial, con un costo de 5,5 mil millones de dólares ${ }^{(10)}$. Están surgiendo otras iniciativas de transporte no motorizado (34), siendo indudable que también deberán afrontarse problemas en esferas conductuales y sociales.

La implementación de la Resolución Directoral $\mathrm{N}$. 6475-2006-MTC/15, Record del Conductor, que crea un Registro Nacional de Sanciones por Infracciones en la cual se consignan obligatoriamente los datos del conductor, licencia e infracciones de tránsito, ha disminuido el número de accidentes. Se debería estudiar si el infractor recurrente tiene actitudes agresivas, prepotentes, impulsividad, tendencia al descontrol o si incurre en consumo de sustancias peligrosas o medicamentos no prescritos $\mathrm{y}$, si están ocurriendo comportamientos violentos, estos deben ser diagnosticados $\mathrm{y}$ atendidos.

La violencia es un fenómeno complejo que puede configurarse como termómetro social e indicador de calidad de vida y que, junto con los accidentes de tránsito, ocasionan más de la mitad de las muertes traumáticas por factores sociales en América Latina (35). Es función de la autoridad regular las relaciones entre los miembros de la comunidad, los responsables no pueden abdicar al mandato que les da la norma ni la población puede renunciar al bienestar y la seguridad.

Se propone que investigadores y universidades contribuyan con estudios multidisciplinarios que aporten a las autoridades los elementos de juicio para identificar y controlar factores como los psicológicos y sociales -violencia en particular- que podrían estar actuando como causales o concurrentes en los accidentes del transporte terrestre.

\section{AGRADECIMIENTOS}

A los doctores Alberto Perales Cabrera y Guillermo Quiroz Jara por sus sugerencias al documento original.

\section{Conflictos de Interés}

Los autores declaran no tener conflictos de interés en la publicación de este artículo.

\section{REFERENCIAS BIBLIOGRÁFICAS}

1. Organización Mundial de la Salud. Informe mundial sobre prevención de los traumatismos causados por el tránsito. Ginebra: OMS; 2004.

2. Banco Interamericano de Desarrollo. Fortalecimiento técnico a la iniciativa de seguridad vial del Banco. Washington DC: BID; 2009.

3. Bancolnteramericanode Desarrollo. Seguridadvial [Página en Internet]. Washington DC: BID; 2009. [fecha de acceso: 28 de mayo de 2010] Disponible en: http://www.iadb.org/ topics $/$ Highways $/$ index.. $\mathrm{cfm}$ ?lang $=$ es\&id $=6872 \&$ artid $=6872$

4. Perú, Ministerio de Salud. Estrategia Nacional de Accidentes de Tránsito [Página en Internet]. Lima: MINSA; 2009 [Fecha de acceso: 25 de marzo de 2010]. Disponible en: http://www. minsa.gob.pe/portada/est_san/accidentes.htm. 
5. Perez-Rincón E, González-Fortaleza C, Ramos L, Jiménes JA. Trastorno por estrés postraumático en pacientes con lesiones no intencionales producidas por accidentes de tránsito. Salud Mental. 2007; 30(6): 43-48.

6. Donroe J, Tincopa M, Gilman RH, Brugge D, Moore DAJ. Pedestrian road traffic injuries in urban Peruvian children and adolescents: case control analyses of personal environmental risk factors. PLoS ONE. 2008; 3(9): e3166.

7. Perú, Ministerio de Salud. Políticas municipales para la promoción de la seguridad vial. Lima: OPS; 2005.

8. Alfaro-Basso D. Problemática sanitaria y social de la accidentalidad del transporte terrestre. Rev Peru Med Exp Salud Publica. 2008; 25(1): 133-37.

9. Defensoría del Pueblo. Informe Defensorial № 137: El transporte urbano en Lima Metropolitana: Un desafío en defensa de la vida. Lima: Defensoría del Pueblo; 2008.

10. Perú, Ministerio de Transportes y Comunicaciones. Plan maestro de transporte urbano para el área metropolitana de Lima y el Callao en la República del Perú. Lima: MTC; 2005.

11. Defensoría del Pueblo. Informe Defensorial № 108. Pasajeros en riesgo: la seguridad en el transporte interprovincial. Lima: Defensoría del Pueblo; 2008.

12. Policía Nacional del Perú. Estadísticas de colisiones viales en el Perú 1998 - 2006. Lima: PNP; 2008.

13. Rey de Castro J, Rosales E, Egoavil M. Somnolencia y cansancio durante la conducción: accidentes de tránsito en las carreteras del Perú. Acta Med Peru. 2009; 29(1): 48-54.

14. Rosales E, Egoavil MT, Durand IS, Montes NE, Flores RE, Rivera SL, et al. Accidentes de carretera y su relación con cansancio y somnolencia en conductores de ómnibus. Rev Med Hered. 20(2): 48-59.

15. Rosales E, Rey de Castro J. Manejar bajo los efectos del alcohol o manejar sin haber dormido adecuadamente, ¿no es lo mismo?. Rev Med Hered. 2009; 20(3): 175-77.

16. Gutierrez JR, Sierra JC, Alfaro 0 . Agresividad al volante en el transporte público de San Salvador: estudio de factores asociados. San Salvador: Universidad Tecnológica de El Salvador; 2008

17. Policía Nacional del Perú. Estadísticas policiales [Página en Internet]. Lima: PNP; 2009. [fecha de acceso: 25 de marzo de 2010] Disponible en: http://www.pnp.gob.pe/ estadistica_policial/estadisticas.asp

18. Espinoza MJ. Violencia en la familia en Lima y el Callao: informe de resultados de la I encuesta de hogares sobre vida familiar en Lima y el Callao. Lima: Ediciones del Congreso del Perú; 2000.

19. Oliveros MA, Figueroa L, Mayorga G, Cano CB, Quispe A, Barrientos A. Violencia escolar (bullying) en colegios estatales de primaria en el Perú. Rev Peru Pediatr. 2008; 61(4): 215-20.

20. García F, Cieza J, Alvarado B. Características de las atenciones registradas por la policía en el servicio de emergencia de un hospital de Lima, 2001. Rev Peru Med Exp Salud Publica. 2005; 22(1): 71-75

21. Rendón L. Prioridades en salud de la Comuna 18 de Cali - Una aproximación hacia la orientación de los servicios de salud. Colombia Med. 2002; 33(2): 58-64.
22. Saavedra-Castillo A. Violencia y salud mental. Acta Med Peru. 2004; 21(1): 39-50.

23. Casanova L, Borges G, Mondragón L, Medina-Mora ME, Cherpitel C. El alcohol como factor de riesgo en accidentes vehiculares y peatonales. Salud Mental. 2001; 24(5): 3-11.

24. De Oliveira AC, Pinheiro JQ. Indicadores psicossociais relacionados a accidentes de trânsito envolvendo motoristas de ônibus. Psicol Estud. 2007; 12(1): 171-78.

25. Ponce C, Bulnes M, Aliaga J, Delgado E, Solís R. Estudio psicológico sobre los patrones de conducta en contextos de tráfico, en grupos de automovilistas particulares y profesionales de Lima Metropolitana. Rev Investig Psicol. 2006; 9(2): 33-64.

26. Centro de Investigación y de Asesoría del Transporte Terrestre. Estudio de casos sobre causas de accidentes de tránsito fatales en Lima Metropolitana y sus probables alternativas de solución. Lima: CIDATT; 2005.

27. Romero C. Manejar en Lima: una aproximación a la cultura ciudadana. Documento en Internet. Lima: PUCP; 2006. [fecha de acceso: 08 de marzo de 2010] Disponible en: http://palestra.pucp.edu.pe/portal/pdf/284.pdf

28. Comisión Nacional del Medio Ambiente/Región Metropolitana. Análisis general del impacto económico y social. Anteproyecto de norma nacional de emisión para vehículos livianos y medianos. Santiago de Chile: CONAMA 2000.

29. Defensoría del Pueblo. Reporte sobre el funcionamiento de los Comités de seguridad ciudadana en el ámbito nacional en el 2009. Lima: Defensoría del Pueblo; 2009.

30. Webb R. Sin reacción. El Comercio. 2010; 12 de abril: a4.

31. Morales-Soto N, Gálvez-Rivero W, Chang-Ausejo C, Alfaro-Basso D, García-Villafuerte A, Ramírez-Maguiña $\mathbf{M}$, et al. Emergencias y desastres: desafíos y oportunidades (de la casualidad a la causalidad). Rev Peru Med Exp Salud Publica. 2008; 25(2): 237-42.

32. Banco Interamericano de Desarrollo. BID lanza plan de acción para mejorar seguridad vial en América Latina y el Caribe [Página en Internet]. Washington DC. BID; 2010. [fecha de acceso: 23 de marzo de 2010] Disponible en: http://www. iadb.org/am/2010/pages.cfm?lang=es\&id=6707\&type=PR

33. Yamada G. Reducción de la pobreza y fortalecimiento del capital social y la participación: La acción reciente del Banco Interamericano de Desarrollo. Washington DC: BID; 2001.

34. Perú, Congreso de la República. Proyecto Ley marco de promoción al uso de la bicicleta como medio de transporte sostenible. Lima: Congreso de la República; 2007.

35. De Souza Minayo MC. Relaciones entre procesos sociales, violencia y calidad de vida. Salud Colectiva. 2005; 1(1): 69-78.

Correspondencia: Dr. Nelson Raul Morales Soto

Dirección: Av. Alameda La Molina Vieja N. ${ }^{\circ} 695$, Dpto. 206. La

Molina, Lima.

Teléfono: (511) 3658891

Correo electrónico: moralessotonelson@gmail.com 